\title{
Oxygen-ozone therapy and local administration induced pain
}

\author{
Marco Mainini, ${ }^{1}$ Maria Laura Rosato, ${ }^{1}$ Margherita Luongo, ${ }^{2}$ Luigi Mascolo, ${ }^{2}$ Silvana Mattera, ${ }^{1}$ \\ Luca Schiaffino ${ }^{2}$ \\ ${ }^{1}$ Department of Anesthesiology, Intensive Care, Pain Medicine and Ozone Therapy, Second University of Naples, Naples; \\ ${ }^{2}$ Center of Oxygen-Ozone Therapy, Master of Oxygen-Ozone Therapy, Second University of Naples, Naples, Italy
}

\begin{abstract}
Oxygen-ozone $\left(\mathrm{O}_{2}-\mathrm{O}_{3}\right)$ therapy is emerging in the treatment of many diseases. This kind of treatment is characterized by a few side effects. When oxygen-ozone mixture is administered through subcutaneous or intramuscular infiltration, often patients refer pain or burning sensation in the site of injection. The aim of our study is to evaluate intensity and duration of this side effect during treatments.
\end{abstract}

\section{Introduction}

Oxygen-ozone $\left(\mathrm{O}_{2}-\mathrm{O}_{3}\right)$ therapy is emerging in the treatment of many diseases, including bone and joint pain conditions (Figure 1). Relatively to these diseases come to our attention a large number of patients suffering from root disks conflict. In these patients, $\mathrm{O}_{2-}$ $\mathrm{O}_{3}$ mixture is administered through subcutaneous and/or intramuscular way. ${ }^{1}$ This administration is carried by a constant burning sensation/pain of variable intensity and duration. ${ }^{2,3}$ The aim of this study is to evaluate the intensity of pain after a local administration of $\mathrm{O}_{2}-\mathrm{O}_{3}$ mixture, through Verbal Rating Scale (VRS).

Correspondence: Marco Mainini, Department of Anesthesiology, Intensive Care, Pain Medicine and Ozone Therapy, Second University of Naples, Naples, Italy.

Email: mamainini@yahoo.it

Key words: Ozone; Local administration; Pain.

Conflict of interest: the authors declare no potential conflict of interest.

Received for publication: 12 April 2017.

Accepted for publication: 12 April 2017.

(C) Copyright M. Mainini et al., 2017

Licensee PAGEPress, Italy

Ozone Therapy 2017; 2:6742

doi:10.4081/ozone.2017.6742

This article is distributed under the terms of the Creative Commons Attribution Noncommercial License (by-nc 4.0) which permits any noncommercial use, distribution, and reproduction in any medium, provided the original author(s) and source are credited.

\section{Materials and Methods}

Patients suffering from disc-articular conflicts are treated through subcutaneous injections and/or intramuscular injections of $\mathrm{O}_{2}-\mathrm{O}_{3}$ mixture of $10 \mathrm{pg}$ in a $10 \mathrm{cc}$ of volume. After the administration of gas all patients feel a sensation or pain of variable duration from 60 seconds to 180 seconds. This happens in 98$100 \%$ of cases at the first dose. In our center, we enrolled a homogeneous group of 30 patients (all suffering from root disk conflict, and aged between 29 and 65 years) that avail themselves of subcutaneous (Figures 2 and 3) and intramuscular $\mathrm{O}_{2}-\mathrm{O}_{3}$ administration. Through Verbal Rating Scale (VRS) we evaluated the intensity of postoperative pain and its duration (Figure 4). VRS is a one-dimensional and categorical scale, which evaluate the intensity of pain through description of the patient, considering four categories (no pain, mild pain, moderate pain, severe pain). We adopted an ozone machine Medical 95 CPS [Multiossigen S.r.l., Gorle (BG), Italy].

\section{Results and Discussion}

At the first treatment, of the 26 patients treated, 21 described severe pain, 4 moderate, 1 mild. In all patients pain after the first treatment lasts less than 120 seconds, in 19 patients less than 90 seconds, in 6 patients less than 40 seconds. From the subsequent treatments, the duration of burning/pain, and the intensity of the same is reduced by $60 \%$ on average.

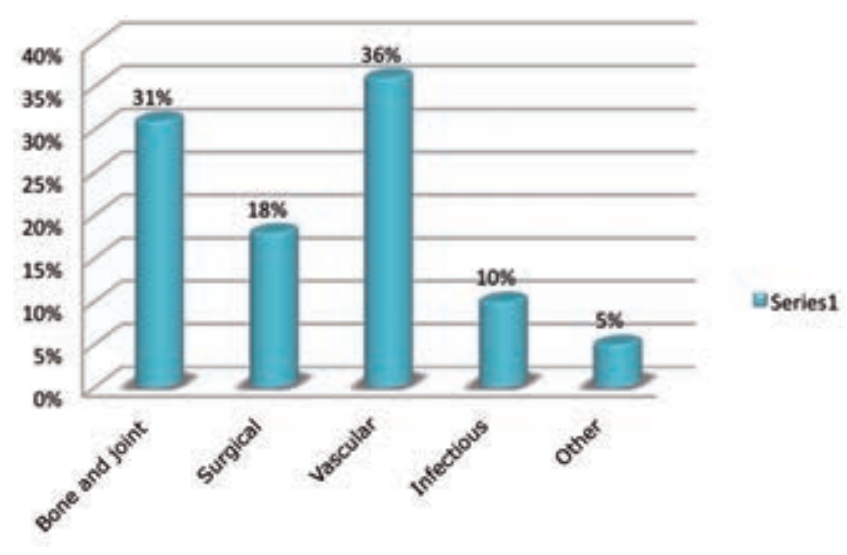

Figure 1. Diseases treated with oxygen-ozone therapy. 


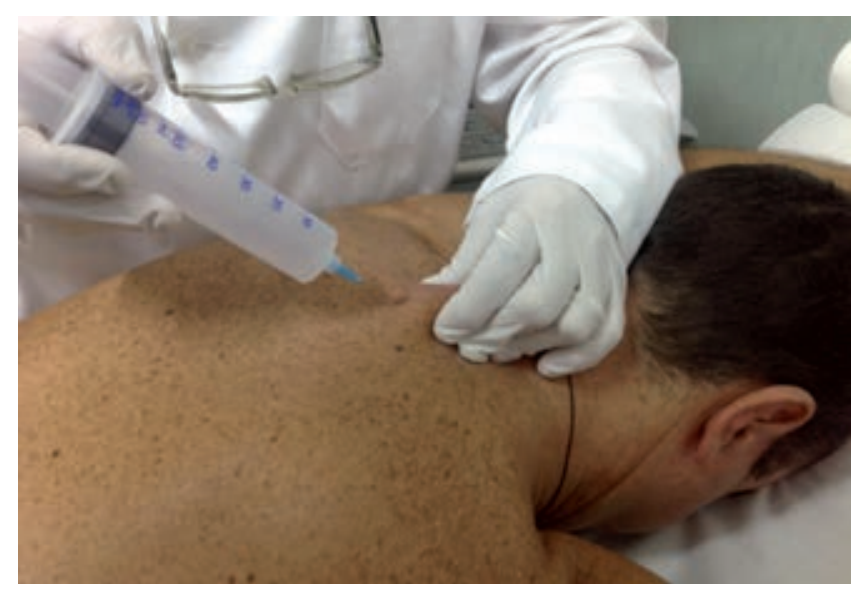

Figure 2. Subcutaneous administration in cervical site.

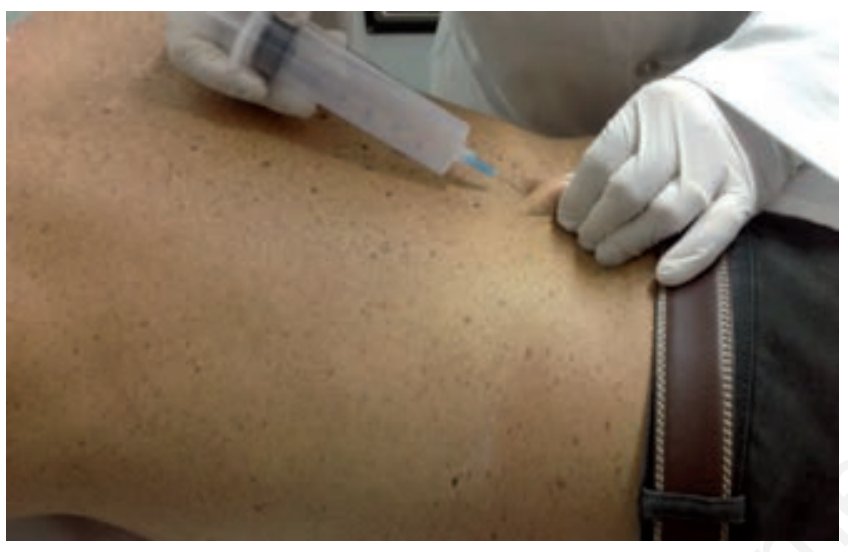

Figure 3. Subcutaneous administration in lumbar site.

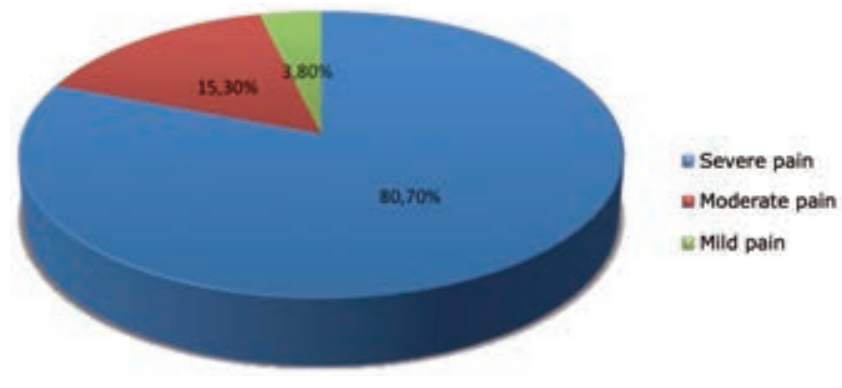

Figure 4. Results expressed as percentage following the Verbal Rating Scale.

\section{Conclusions}

$\mathrm{O}_{2}-\mathrm{O}_{3}$ administration of causes pain in the injection site in all treated patients. This pain is described as severe by $80.7 \%$ of patients, moderate in $15.3 \%$, mild in $3.8 \%$ of patients. It has a duration of less than 3 minutes in $100 \%$ of the same. The duration is reduced of $60 \%$ in the subsequent treatments.

\section{References}

1. Bocci V. Scientific and medical aspects of ozone therapy. State of the art. Arch Med Res 2006;37:425-35.

2. Chiefari M, Verde N, Salerno N, et al. Valutazione antalgica dell'ozonoterapia. Acta Toxicol Terap 1996;17:253-6.

3. Salerno N, Mascolo L, Morsicano A, et al. Antalgic effect of ozone after local administration. PRAP 1996. 